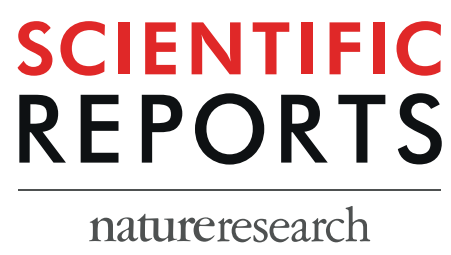

Received: 14 March 2019

Accepted: 27 July 2019

Published online: 07 August 2019

\title{
Beta-blocker choice and
} exchangeability in patients with heart failure and chronic obstructive pulmonary disease: an
Italian register-based cohort study Maurizio Sessa $\mathbb{1}^{1,2}$, Annamaria Mascolo2 , Daniel Bech Rasmussen ${ }^{3,4}$, Kristian Kragholm ${ }^{5}$, Magnus Thorsten Jensen ${ }^{6}$, Liberata Sportiello ${ }^{2}$, Concetta Rafaniello ${ }^{2}$, Giuseppe Michele Tari ${ }^{7}$, Claudia Pagliaro 7 , Morten Andersen $\mathbb{1}^{1}$, Francesco Rossi ${ }^{2} \&$ Annalisa Capuano ${ }^{2}$

Clinical guidelines suggest that for patients with heart failure and concurrent chronic obstructive pulmonary disease (COPD), metoprolol/bisoprolol/nebivolol should be preferred over carvedilol. However, studies suggest a high proportion of carvedilol usage that remains unexplained. Therefore, we aimed to investigate the predictors of carvedilol choice in patients with heart failure and COPD that were naïve to carvedilol or metoprolol/bisoprolol/nebivolol. Caserta Local Health Unit databases (Italy) were used as data sources. Age, sex, chronic/acute comorbidities, and co-medications were included in a logistic regression model to assess predictors of carvedilol choice. Chronic comorbidities include those defined in the Elixhauser comorbidity index and all hospitalizations within two years prior to the first beta-blocker prescription. Comedications include all redeemed prescriptions within one year prior to the beta-blocker prescription. Kernel density estimations were used to assess the overlap in propensity and preference scores distributions for receiving carvedilol and thereby potential beta-blocker exchangeability. Totally, 10091 patients composed the study population; 2011 were exposed to carvedilol. The overlapping of propensity scores distributions was $57 \%$. Accordingly, the exchangeability was not reached. Atrioventricular block (Odds Ratio, OR 8.20; 95\% Confidence Interval, 95\% CI 1.30-51.80), cerebrovascular thrombosis (OR 7.06; 95\% CI 1.14-43.68), chronic kidney disease (OR 4.32; 95\% Cl 1.16-16.02), and acute heart failure (OR 1.97; 95\% Cl 1.28-3.03) hospitalizations were statistically significantly associated with carvedilol choice. Analogously, human insulin (OR 3.00; 95\% Cl 1.24-7.24), fondaparinux (OR 2.47; 95\% Cl 1.17-5.21) or strontium ranelate (OR 2.03; $95 \% \mathrm{Cl} 1.06-3.90)$ redeemed prescriptions. In conclusion, this study suggests the absence of betablockers exchangeability and a preferential choice of carvedilol in patients with heart failure, COPD and concurrent chronic kidney disease, atrioventricular block, cerebrovascular thrombosis, acute heart failure or redeeming human insulin, fondaparinux or strontium ranelate prescriptions. Therefore, it suggests that choice of prescribing carvedilol over metoprolol/bisoprolol/nebivolol is driven by differences in comorbidities and co-treatments.

\footnotetext{
${ }^{1}$ Department of Drug Design and Pharmacology, University of Copenhagen, Jagtvej 160, 2100, København $\varnothing$, Denmark. ${ }^{2}$ Campania Pharmacovigilance and Pharmacoepidemiology Regional Centre, Department of Experimental Medicine, University of Campania "L. Vanvitelli", Via Santa Maria di Costantinopoli 16, 80138, Naples, Italy. ${ }^{3}$ Respiratory Research Unit Zealand, Department of Respiratory Medicine, Naestved Hospital, Ringstedgade 61, 4700, Næstved, Denmark. 'Department of Cardiology, Herlev and Gentofte University Hospital, Gentofte Hospitalsvej 1, 2900, Hellerup, Denmark. ${ }^{5}$ Unit of Epidemiology and Biostatistics, Aalborg University Hospital, Hobrovej 18-22, 9100, Aalborg, Denmark. ${ }^{6}$ Heart Centre, Copenhagen University Hospital Rigshospitalet, Blegdamsvej 9, 2100, København, Denmark. ${ }^{7}$ Caserta Local Health Unit, Via Unità Italiana 28, 81100, Caserta, Italy. Correspondence and requests for materials should be addressed to M.S. (email: maurizio.sessa@sund.ku.dk)
} 
Beta-blockade is a crucial pharmacological therapy to improve survival and to reduce morbidity in patients with heart failure and concurrent chronic obstructive pulmonary disease ${ }^{1}$. The benefits of beta-blockade in heart failure are due to their mitigation of the negative pathological consequences associated with adrenergic nervous system hyperactivity that gradually occurs in a chronically failing heart ${ }^{2}$. The sympathetic overdrive in heart failure is associated with an increased risk of arrhythmias, progressive dysfunction of the left ventricle, and myocardial insult, phenomena that have a negative impact on disease progression and prognosis ${ }^{3}$. In fact, adrenergic nervous system hyperactivity determines increased peroxidative and lipoperoxidative metabolism and demands of oxygen in the myocardium leading to the overproduction of reactive oxygen species that in turn determines local inflammation, apoptosis, and necrosis resulting in interstitial fibrosis and heart remodelling ${ }^{4,5}$. In Europe, four beta-blockers are currently indicated for heart failure, three beta-1 adrenoceptor selective, metoprolol, bisoprolol and nebivolol, and a non-selective, carvedilol. Metoprolol and bisoprolol are both second generation beta-blockers or rather they have high beta-1 adrenoceptor selectivity and they both lack intrinsic sympathomimetic activity. Bisoprolol has 20 times higher selectivity for the beta-1 adrenoceptor than metoprolol and it has a longer half-life (9-12 hours versus 3-7 hours) and bioavailability (90 versus 12\%). Due to its high selectivity for the beta-1 adrenoceptor, bisoprolol has minimal effects on insulin sensitivity while, on the other hand, metoprolol has an additional membrane-stabilizing activity on the heart. Carvedilol and nebivolol are third generation beta-blockers with additional vasodilatation properties. Nebivolol is beta-1 adrenoceptor selective beta-blockers with a long half-life and bioavailability (12-96\%) which, through a mechanism mediated by nitric oxide, is able to provide a reduction of the arteries tone that when targeted on the coronaries guarantees additional cardioprotective and anti-ischemic effects. Carvedilol is a non- beta-1 adrenoceptor selective beta-blockers with an intrinsic sympathomimetic activity, long half-life (7-10 hours) and additional vasodilatation properties mediated by the alpha-1 adrenoceptor blockade. It has anti-oxidant properties, it mitigates calcium overload by modulating ryanodine channels, and it ameliorates insulin sensitivity ${ }^{6}$. Despite a clinical and pharmacological rationale suggest the use of beta-blockers in heart failure, reluctance to prescribe beta-blockers in patients with the concurrent chronic obstructive pulmonary disease has been observed in multiple clinical settings. The leading factor for under-prescription of beta-blockers in this subpopulation is the concern of adverse respiratory effects mediated by beta-blockers through beta- 2 adrenoceptors in the airway. The magnitude of the bronchoconstriction is strongly correlated with beta- 1 adrenoceptor selectivity ${ }^{7}$. For this reason, current clinical guidelines suggest that for patients with heart failure and concurrent chronic obstructive pulmonary disease, metoprolol, bisoprolol or nebivolol should be preferred ${ }^{8,9}$. However, studies suggest a high proportion of carvedilol usage that is in opposition to above-mentioned recommendations ${ }^{10}$. Several adverse clinical consequences have been associated with carvedilol usage in patients with heart failure and concurrent chronic obstructive pulmonary disease such as a reduction in the lung function ${ }^{11}$ that may lead to subclinical effects on respiratory parameters ${ }^{10,12,13}$. Additionally, carvedilol may lead to a higher rate of unfavourable hemodynamic alterations leading to an increased risk of heart failure hospitalization, on both short and long-term following treatment commencement ${ }^{10,12}$. In virtue of the potential negative clinical consequences associated with carvedilol choice in patients with heart failure and chronic obstructive pulmonary disease naïve to beta-blockers, understanding key factors that may lead to a preferential choice of such prescriptions is crucial and, in this regard, the evidence is scarce. Our hypothesis is that concurrent chronic/acute comorbidities and comedications may have driven the choice of carvedilol over metoprolol/bisoprolol/nebivolol and this may have led to the absence of exchangeability of these beta-blockers in this subpopulation. To overcome this gap in knowledge, the current study aims to investigate carvedilol and metoprolol/bisoprolol/nebivolol exchangeability and the predictors of carvedilol choice in patients with heart failure and chronic obstructive pulmonary disease that were naïve to beta-blockers. The investigated predictors included chronic comorbidities defined in the Elixhauser comorbidity index ${ }^{14}$, all hospitalizations and redeemed prescriptions within two and one years prior to the first beta-blocker prescription, respectively.

\section{Methods}

Data sources. Health data sources of the Caserta Local Health Unit were used to identify the study population. These data sources cover approximately 1,000,000 citizens living in the catchment area of Caserta (Campania Region, Italy). For reimbursement purposes, the data sources store information on socio-demographic characteristics of residents, redeemed prescriptions, inpatient and outpatient hospitalizations and hospital contacts. Data contained in Caserta Local Health Unit administrative databases are pseudonymized to comply with current law on data protection and privacy.

Study population. The study population included patients who (1) during the period December 31, 2010 to September 30, 2017 had a hospitalization/hospital contact for heart failure, (2) redeemed their first carvedilol, metoprolol, bisoprolol or nebivolol prescription after this hospitalization for heart failure (at least one year wash-out period for the beta-blocker prior to the heart failure hospitalization), and (3) redeemed drugs for obstructive airway diseases (Anatomical Therapeutic Chemical Classification, ATC R03) prior to the redemption of the first prescription of aforementioned beta-blockers (Fig. 1). In Italy, drugs for obstructive airway disease (ATC R03) that are reimbursed by the Italian Healthcare system are indicated for asthma or chronic obstructive pulmonary disease ${ }^{12}$. However, the use of beta-blockers in asthma is relatively contraindicated in the summaries of product characteristics and is not recommended in current clinical guidelines. Therefore, we believe that by using this approach we identified mostly patients with chronic obstructive pulmonary disease.

Study outcome. The study outcome was the probability of redeeming a carvedilol as opposed to metoprolol/ bisoprolol/nebivolol prescription. 


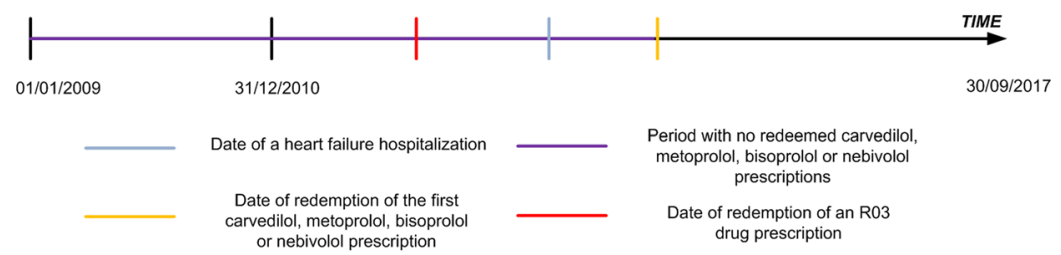

Figure 1. Example of the temporal sequence of events needed for patients to be included in the study population.

Potential predictors for carvedilol choice. Age, gender, year of inclusion in the study population and chronic comorbidities according to the Elixhauser Comorbidity Index were included as potential predictors $^{14}$. Chronic comorbidities were identified by hospital discharge codes prior to the redemption of the first beta-blocker prescription. Other potential predictors included all primary diagnosis for hospital contact/hospitalization and redeemed prescriptions that occurred 2 and 1 year/s prior to the redemption of the first carvedilol, metoprolol, bisoprolol, or nebivolol prescription, respectively.

Statistical analyses. A prevalence proportion (the prevalence of the event among patients exposed to carvedilol divided by the prevalence of the event among patients exposed to metoprolol, bisoprolol, or nebivolol) for all primary diagnosis for hospital contact/hospitalization and redeemed prescriptions that occurred 2 and 1 year/s prior to the redemption of the first carvedilol, metoprolol, bisoprolol, or nebivolol prescription was computed and the 500 event most prevalent among carvedilol users were selected. This is a crucial preliminary step to identify the most relevant variables for propensity score assessment in highly dimensional datasets. The 500 top-ranked potential predictors selected with the abovementioned method were used as independent variables in a logistic regression model to compute the propensity score for receiving carvedilol rather than metoprolol, bisoprolol, and nebivolol. The predicted probability of receiving carvedilol given the set of potential predictors was used to assess the exchangeability between treatments groups given their covariate distributions. The null hypothesis was the absence of clinical or demographic differences between recipients of carvedilol versus metoprolol, bisoprolol, or nebivolol or rather beta-blocker exchangeability (referred to as empirical 'equipoise' in Greenland et al.) ${ }^{15}$. According to Walker and colleagues, the predicted value from the above-mentioned logistic regression was used to compute a preference score as following: $\ln \{A /(1-A)\}=\ln (B /(1-B)-\ln (C /(1-C))$ where $A$ is the preference score, $\mathrm{B}$ is the propensity score and $\mathrm{C}$ is the prevalence of exposure to carvedilol ${ }^{16}$. To assess beta-blocker exchangeability among beta-blockers, a Kernel density estimation was used to assess the overlap in propensity scores areas for carvedilol and metoprolol/bisoprolol/nebivolol users. Additionally, as suggested by Walker and colleagues, by using a Kernel density estimation, we tested if half of the distributions of the preference score was between 0.3 and 0.7 between carvedilol and metoprolol/bisoprolol/nebivolol users ${ }^{16}$. Baseline characteristics of the two cohorts were compared using the $t$-tests for continuous variables and $\chi^{2}$ test for categorical variables.

Compliance with ethical standards. According to Italian National laws, ethical approval or informed consent for register-based studies is not required.

\section{Results}

The study population's demographic and clinical characteristics are provided in Table 1. In total, 10091 patients were included in the study population of which 2011 (19.9\%) exposed to carvedilol. The mean age was 77.7 years (standard deviation of 11.8 years).

Beta-blocker exchangeability in patients with heart failure and concurrent chronic obstructive pulmonary disease. The estimated overlapping area of two kernel density estimations of propensity scores among treated with carvedilol and metoprolol/bisoprolol/nebivolol was 57\% (Fig. 2). The exchangeability among beta-blockers according to the preference score criteria was not reached (Fig. 3). Both results suggest the absence of exchangeability between treatment groups and therefore significant differences in comorbidities/ co-treatments distributions among the 500 top-ranked potential predictors between patients receiving carvedilol over metoprolol/bisoprolol/nebivolol.

Predictors of carvedilol choice. Odds ratios and 95\% confidence intervals (95\% CI) of statistically significant predictors for carvedilol prescription over metoprolol/bisoprolol/nebivolol prescriptions are provided in Fig. 4. Age, gender, and comorbidities according to the Elixhauser Comorbidity Index were not predictors of carvedilol choice. Among the 500 top-ranked potential predictors, atrioventricular block (OR 8.20; 95\% CI 1.3051.80), cerebrovascular thrombosis (OR 7.06; 95\% CI 1.14-43.68), chronic kidney disease (OR 4.32; 95\% CI 1.1616.02), and acute heart failure (OR 1.97; 95\% CI 1.28-3.03) hospitalizations/hospital contacts within two years prior to the first beta-blocker prescription were associated with carvedilol choice. Analogously, patients redeeming a prescription of human insulin, fondaparinux or strontium ranelate within one year prior to the beta-blocker prescription had significantly higher odds of receiving carvedilol over metoprolol/bisoprolol/nebivolol. 


\begin{tabular}{|c|c|c|c|c|}
\hline Variable & $\begin{array}{l}\text { Metoprolol/Bisoprolol/ } \\
\text { Nebivolol }(\mathbf{n}=\mathbf{8 0 8 0})\end{array}$ & $\begin{array}{l}\text { Carvedilol } \\
(\mathbf{n}=2011)\end{array}$ & $\begin{array}{l}\text { Total } \\
(\mathrm{n}=10091)\end{array}$ & p-value \\
\hline Age & $77.8(11.7)$ & $77.7(11.9)$ & $77.8(11.8)$ & 0.922 \\
\hline Sex (male) & $4092(50.7)$ & $970(48.2)$ & $5062(50.2)$ & 0.079 \\
\hline Pulmonary embolism & $77(1.0)$ & $16(0.8)$ & $93(0.9)$ & 0.595 \\
\hline Peripheral arterial disease & $385(4.8)$ & $88(4.4)$ & $473(4.7)$ & 0.496 \\
\hline Liver disorders & $400(5.0)$ & $88(4.4)$ & $488(4.8)$ & 0.309 \\
\hline Cancer & $1248(15.4)$ & $306(15.2)$ & $1554(15.4)$ & 0.825 \\
\hline Chronic kidney disease & $526(6.5)$ & $123(6.1)$ & $649(6.4)$ & 0.553 \\
\hline Arterial embolism & $1640(20.3)$ & $373(18.5)$ & $2013(19.9)$ & 0.084 \\
\hline Diabetes mellitus type 2 & $1698(21.0)$ & $420(20.9)$ & $2118(21.0)$ & 0.922 \\
\hline Hypertension & $2766(34.2)$ & $679(33.8)$ & $3445(34.1)$ & 0.711 \\
\hline Acute myocardial infarction & $1214(15.0)$ & $290(14.4)$ & $1504(14.9)$ & 0.518 \\
\hline Atrial fibrillation & $343(4.2)$ & $88(4.4)$ & $431(4.3)$ & 0.842 \\
\hline Ventricular fibrillation & $423(5.2)$ & $104(5.2)$ & $527(5.2)$ & 0.953 \\
\hline Valvular Disease & $291(3.6)$ & $70(3.5)$ & $361(3.6)$ & 0.846 \\
\hline $\begin{array}{l}\text { Pulmonary Circulation } \\
\text { Disorders }\end{array}$ & $94(1.2)$ & $23(1.1)$ & $117(1.2)$ & 1.000 \\
\hline Depression & $56(0.7)$ & $17(0.8)$ & $73(0.7)$ & 0.565 \\
\hline Alcohol Abuse & $46(0.6)$ & $3(0.1)$ & $49(0.5)$ & 0.024 \\
\hline Obesity & $240(3.0)$ & $85(4.2)$ & $325(3.2)$ & 0.005 \\
\hline Coagulopathy & $30(0.4)$ & $6(0.3)$ & $36(0.4)$ & 0.778 \\
\hline Rheumatoid Arthritis & $24(0.3)$ & $10(0.5)$ & $34(0.3)$ & 0.241 \\
\hline $\begin{array}{l}\text { Solid Tumour without } \\
\text { Metastasis }\end{array}$ & $64(0.8)$ & $18(0.9)$ & $82(0.8)$ & 0.747 \\
\hline Lymphoma & $15(0.2)$ & $4(0.2)$ & $19(0.2)$ & 1.000 \\
\hline
\end{tabular}

Table 1. Demographic and clinical characteristics of patients with heart failure and chronic obstructive pulmonary disease that received for the first time a prescription of carvedilol, metoprolol, bisoprolol or nebivolol and that were resident in the catchment area of Caserta Local Health Unit (Campania Region, Italy).

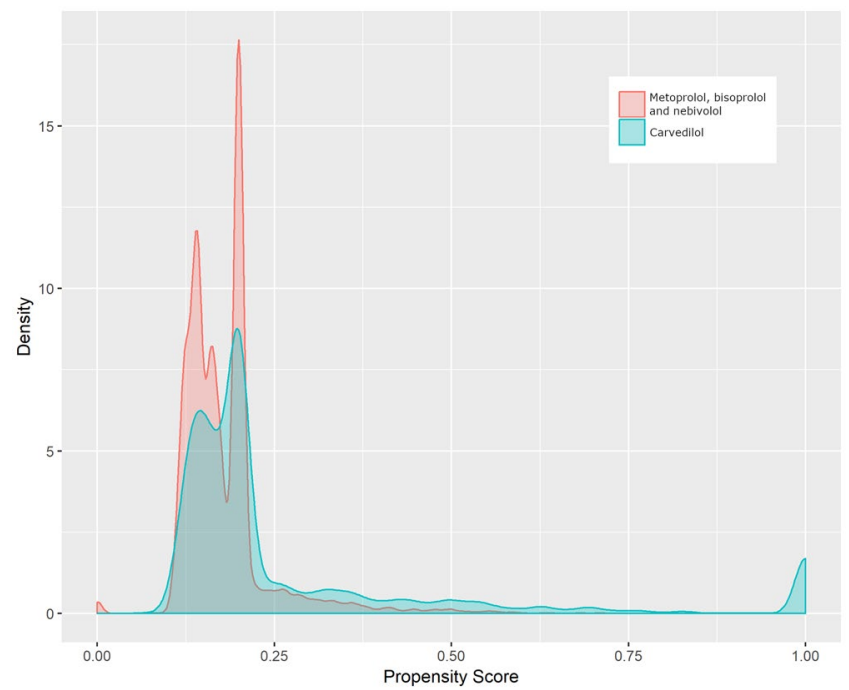

Figure 2. Kernel density estimations of propensity score among patients treated with carvedilol and metoprolol/bisoprolol/nebivolol having heart failure and chronic obstructive pulmonary disease located in the catchment area of Caserta Local Health Unit (Campania Region, Italy).

\section{Discussion}

This study suggests that among patients with heart failure and chronic obstructive pulmonary disease, the choice of prescribing carvedilol over metoprolol/bisoprolol/nebivolol is driven by differences in comorbidities and co-treatments. Predictors of carvedilol choice were chronic comorbidities and co-treatments including chronic kidney disease, human insulin, fondaparinux, strontium ranelate or recent acute episode of acute heart failure, cerebrovascular thrombosis or atrioventricular block. 


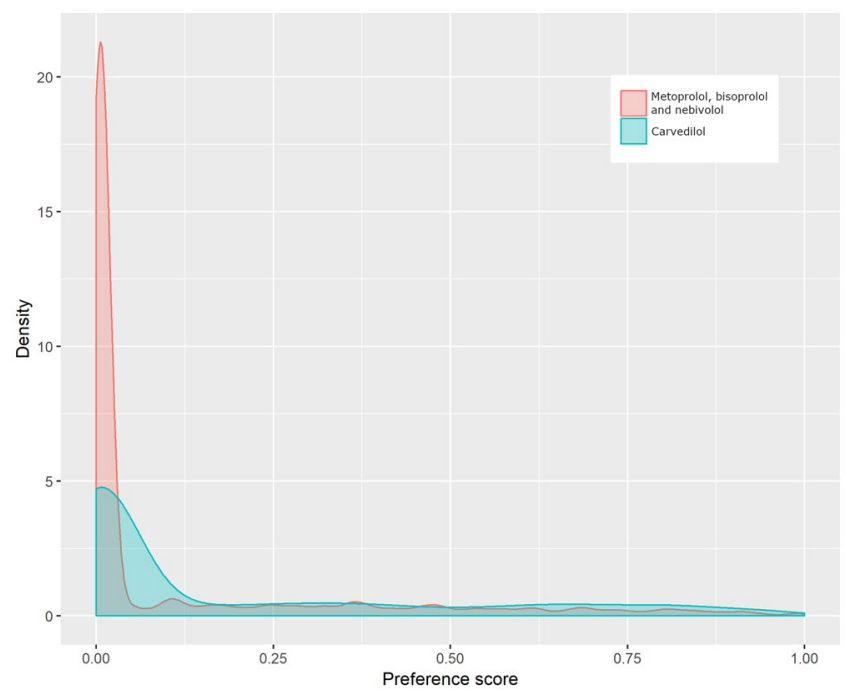

Figure 3. Kernel density estimations of preference score among patients treated with carvedilol and metoprolol/bisoprolol/nebivolol having heart failure and chronic obstructive pulmonary disease located in the catchment area of Caserta Local Health Unit (Campania Region, Italy).

Explanatory variable

Atrioventricular block

Cerebrovascular thrombosis

Chronic kidney disease - stage 2

Fondaparinux

Insulin (human)

Strontium ranelate

Acute heart failure

$\begin{array}{rr}\text { Estimate } & \mathbf{9 5 \%} \mathbf{C I} \text { low } \\ 8.20 & 1.30 \\ 7.06 & 1.14 \\ 4.32 & 1.16 \\ 2.47 & 1.17 \\ 3.00 & 1.24 \\ 2.03 & 1.06 \\ 1.97 & 1.28\end{array}$

$\begin{array}{rr}\text { 95\%CI high } & \text { P-value } \\ 51.80 & 0.025 \\ 43.68 & 0.036 \\ 16.02 & 0.029 \\ 5.21 & 0.018 \\ 7.24 & 0.015 \\ 3.90 & 0.034 \\ 3.03 & 0.002\end{array}$

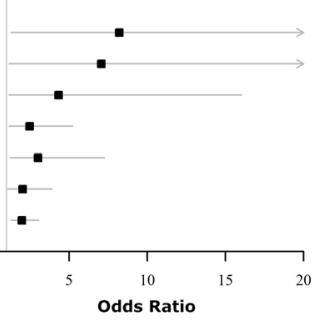

Figure 4. Predictors of carvedilol prescription over metoprolol/bisoprolol/nebivolol for patients with heart failure and chronic obstructive pulmonary disease located in the catchment area of Caserta Local Health Unit (Campania Region, Italy).

European clinical guidelines on chronic heart failure released during the study period do not state any preference among beta-blockers in the aforementioned clinical conditions and they do not provide recommendations in the clinical setting of multiple comorbidities. However, clinical evidence seems to support the choice of carvedilol over other beta-blockers in the clinical scenario listed above.

Carvedilol was found beneficial for patients with chronic kidney disease due to its ability to reduce cardiac output and renin release, as to its ability to reduce renovascular constriction through its alfa- 1 and beta- 1 receptor antagonism. Additional, carvedilol was useful due to its enhanced renovascular dilation through beta2-receptor antagonism ${ }^{17}$. These effects, along with to the ability of all beta-blockers in reducing sympathetic over-activity, were found associated with attenuation in the increase of albuminuria and hyperkalaemia, but also to an absence of an increase in serum creatinine, events typically observed in patients with chronic kidney disease. In addition, the aforementioned effects were found associated with high effectiveness in protecting from cardiovascular events in patients with chronic kidney disease ${ }^{17-19}$.

With regards to diabetes mellitus and in particular human insulin as a predictor for carvedilol choice, evidence suggests a favourable effect of carvedilol on glycaemic control in diabetic patients when compared to metoprolol. In particular, in the Glycaemic Effects in Diabetes Mellitus: Carvedilol-Metoprolol Comparison in Hypertensive (GEMINI) trial, patients treated with carvedilol had a lower probability of new onset diabetes mellitus, microalbuminuria (a surrogate marker for endothelial function) and an increased insulin sensitivity compared to patients treated with metoprolol. Additionally, a recent systematic review suggested even more beneficial effect of carvedilol in diabetes mellitus, including a reduced risk of new onset of atherogenic dyslipidaemia or weight gain among treated with carvedilol ${ }^{20,21}$.

Regarding the role of strontium ranelate as a predictor for carvedilol choice, it should be mentioned that our study population had a mean age of 77.8 years with a proportion of women of $49.8 \%$ and, therefore, at high risk of osteoporosis. Additionally, patients with chronic obstructive pulmonary disease have an increased risk of all-cause and site-specific fractures ${ }^{22}$. In Italy, during the study period, strontium ranelate was indicated for "the treatment of severe osteoporosis in postmenopausal women and in adult men at high risk of fracture". Observational studies suggest an association between beta-blocker treatments, and in particular carvedilol, and reduced risk of bone fracture and an inherent protective effect of this drug class. On the other hand, pooled data from clinical 
trials that investigate the effect of carvedilol on congestive heart failure do not seem to support this hypothesis ${ }^{23,24}$. Mechanistic studies performed in pre-clinical models support the hypothetic involvement of $\beta$-adrenoceptor in the aforementioned phenomena by showing that beta-blocker treatment improves bone density and mass while $\beta$-adrenoceptor agonists led to the opposite effect. In particular, the effect seems mediated by the $\beta 2$-adrenoceptor on osteoblasts and the sympathetic nervous system ${ }^{25}$. It should be mentioned that no formal potential drug-drug interactions were found between strontium ranelate and carvedilol or metoprolol/bisoprolol/nebivolol by using Micromedex ${ }^{\circledR 26}$. Therefore we excluded potential drug-drug interaction as a plausible explanation for carvedilol choice in these patients.

In relation to fondaparinux as a predictor of carvedilol prescription, we need to mention that during the study period, fondaparinux was indicated for the prevention of venous thromboembolism in multiple clinical scenarios. We believe that a plausible explanation for the higher proportion of fondaparinux use in patients treated with carvedilol may be due to the fear of potential drug-drug interactions. In particular, clinical guidelines advocate the use of fondaparinux for venous thromboembolism prophylaxis in patients with a history of heparin-induced thrombocytopenia ${ }^{27}$. Alternatives to fondaparinux in the aforementioned clinical scenario are the direct acting oral anticoagulants ${ }^{27}$. However, while carvedilol has no potential drug-drug interactions with fondaparinux ${ }^{26}$, it has with direct acting oral anticoagulants, and in particular dabigatran and edoxaban. Given carvedilol's ability to inhibit the glycoprotein-P, it may induce an increased plasma concentration of the aforementioned direct acting oral anticoagulants with a consequent increased risk of bleeding ${ }^{26}$. Therefore, we speculate that the occurrence of these clinical scenarios may have led the choice of carvedilol in patients exposed to fondaparinux.

In relation to acute heart failure, previous studies have found different effects on the cardiovascular system among beta-blockers with different $\beta$ - 1 adrenoceptor selectivity in patients that experienced acute heart failure and that were exposed to inotropic agents (i.e. dobutamine). In this subpopulation, metoprolol did not modify the cardiac index, heart rate, and systemic vascular resistance providing only a modest effect on the pulmonary artery and systemic arterial pressures within the first 12 months of treatment. In contrast, substantial differences were observed for carvedilol. The aforementioned effects were attributed to the blockade of $\beta$-2-receptors in the vascular system and to the other pharmacological properties of carvedilol ${ }^{28,29}$.

It should be mentioned that clinical evidence exists on the beneficial effect of beta-blockers on the risk of early death in patients with ischemic stroke ${ }^{30}$. In pre-clinical and in-vitro models, authors found neuroprotective effects of beta-blockers with additional vasodilatation properties, like carvedilol and nebivolol. Notably, for carvedilol, a study suggests that these effects may be mediated also by the $\beta 2$ adrenal receptors antagonism and cyclooxygenase 2 isozyme/prostacyclin pathways ${ }^{31,32}$. Additionally, clinical evidence seems to support the hypothesis of a reduced risk of mortality due to stroke in patients exposed to carvedilol versus those exposed to metoprolol ${ }^{33}$.

Regarding the choice of carvedilol in patients with a recent episode of atrioventricular block, it should be highlighted that clinically relevant differences were observed among beta-blockers indicated for heart failure on the heart rate. In particular, among aforementioned beta-blockers, carvedilol provided a weaker effect on heart rate at rest when compared to other beta-blockers especially in patients with a low sympathetic tone which may be beneficial in patients with a recent episode of atrioventricular block. It was hypothesized that this effect might be caused by the increased sympathetic drive consequent to the peripheral vasodilation resulting from the accessories vasodilatation properties of carvedilol ${ }^{34,35}$.

Strengths and limitations. A major limitation of this study is the lack of information on the indication of use for redeemed prescriptions. For drugs having ATC code R03, it may have led to a misclassification of the exposure. In fact, these drugs are mainly indicated for both asthma and chronic obstructive pulmonary diseases. Therefore, we may have classified patients with asthma as having chronic obstructive pulmonary disease. We believe that the magnitude of this type of misclassification is very low because Caserta Local Health unit databases contain information on drugs reimbursed by the national health system, and most of the reimbursed drugs in this ATC group are indicated for chronic obstructive pulmonary disease as shown elsewhere ${ }^{36}$. Additionally, beta-blockers are relatively contraindicated in asthma.

The second major limitation of this study is the lack of information on over-the-counter drugs/drugs not requiring a medical prescription. In particular, we may have not detected predictors for carvedilol choice among acute comorbidities. In the Italian healthcare system, over-the-counter drugs/drugs not requiring a medical prescription are generally not indicated for chronic and/or invalidating diseases with a high burden for the patient and they are usually used as symptomatic treatments ${ }^{37}$. Another limitation of this study is the potential misclassification of chronic comorbidities identified through hospitalizations/hospital contacts. In fact, we lack information for those patients hospitalized and/or with a hospital contact outside the catchment area of Caserta local health unit. However, we believe that the magnitude of this bias is low because these patients will receive in most of the cases a pharmacological treatment that is tracked in Caserta Local health unit databases.

The third major limitation of this study is the lack of information on the ethnicity/race of the study population for which, recent studies found an association with a differential response to beta-blockers treatment ${ }^{38,39}$. However, we believe that this limitation may have introduced a negligible bias. In fact, during the study period, the number of immigrants in the catchment area of Caserta was between 3000-4000, or rather $0.3-0.4 \%$ of the study population and, mostly with an age lower than 60 years and, therefore, with a lower probability of having heart failure ${ }^{40}$.

Regarding the lack of information on the cytochrome P450 2D6 polymorphisms in the study population, despite carvedilol is extensively metabolized by this enzyme ${ }^{41,42}$, to date, in Europe, none of the summaries of product characteristics of medicinal products containing carvedilol include polymorphisms of the gene codifying this enzyme as a "contraindications"/"special warnings and precautions for use" for its prescription. Therefore, we believe that even in the setting of poor, rapid or ultra-rapid metabolizers, it should not have influenced the prescription choice. 
Finally, as for all observational studies, we cannot exclude the presence of unmeasured confounders that may have introduced biases in our analysis. Strengths include the use of the entire population of patients enlisted in Caserta local health unit databases reducing the risk of selection bias and the possibility of providing results based on real-world data from routine clinical practice.

\section{Conclusion}

In conclusion, this study showed that no beta-blocker exchangeability was found among carvedilol and metoprolol/bisoprolol/nebivolol in patients with heart failure and chronic obstructive pulmonary disease. In this subpopulation, concurrent chronic (i.e. chronic kidney disease) and acute comorbidities (i.e. acute heart failure, cerebrovascular thrombosis or atrioventricular block) or concurrent pharmacological treatments (i.e. fondaparinux, human insulin and strontium ranelate) may drive the choice of prescribing carvedilol over metoprolol/bisoprolol/nebivolol.

\section{Data Availability}

The datasets generated during and/or analyzed during the current study are not publicly available due to legal reservations but are available from the corresponding author on reasonable request.

\section{References}

1. Sin, D. D., Anthonisen, N. R., Soriano, J. B. \& Agusti, A. G. Mortality in COPD: Role of comorbidities. Eur. Respir. J. 28, 1245-1257 (2006).

2. Lymperopoulos, A. Physiology and pharmacology of the cardiovascular adrenergic system. Front. Physiol. 4, 240 (2013).

3. Santulli, G. \& Iaccarino, G. Adrenergic signaling in heart failure and cardiovascular aging. Maturitas 93, 65-72 (2016).

4. Hatton, R., Cvjeticanin, A. \& Lymperopoulos, A. The adrenergic system of the adrenal glands as a remote control of cardiac function. J Cardiovasc Dis 5, 394-397 (2015).

5. Ciccarelli, M., Sorriento, D., Coscioni, E., Iaccarino, G. \& Santulli, G. Adrenergic receptors. In Endocrinology of the Heart in Health and Disease 285-315 (Elsevier, 2017).

6. Santulli, G. Sympathetic Nervous System Signaling in Heart Failure and Cardiac Aging BT - Pathophysiology and Pharmacotherapy of Cardiovascular Disease. in (eds Jagadeesh, G., Balakumar, P. \& Maung-U, K.) 83-105, https://doi.org/10.1007/978-3-319-159614_5 (Springer International Publishing, 2015).

7. Lim, K. P., Loughrey, S., Musk, M., Lavender, M. \& Wrobel, J. P. Beta-blocker under-use in COPD patients. Int. J. Chron. Obstruct. Pulmon. Dis. 12, 3041-3046 (2017).

8. Mirza, S., Clay, R. D., Koslow, M. A. \& Scanlon, P. D. COPD Guidelines: A Review of the 2018 GOLD Report. Mayo Clin. Proc. 93, 1488-1502 (2018).

9. Ponikowski, P. et al. 2016 ESC Guidelines for the diagnosis and treatment of acute and chronic heart failure: The Task Force for the diagnosis and treatment of acute and chronic heart failure of the European Society of Cardiology (ESC)Developed with the special contribution of. Eur. Heart J. 37, 2129-2200 (2016).

10. Sessa, M. et al. Relationship between heart failure, concurrent chronic obstructive pulmonary disease and beta-blocker use: a Danish nationwide cohort study. Eur. J. Heart Fail. 20, 548-556 (2018).

11. Jabbour, A. et al. Differences between beta-blockers in patients with chronic heart failure and chronic obstructive pulmonary disease: a randomized crossover trial. J. Am. Coll. Cardiol. 55, 1780-1787 (2010).

12. Sessa, M. et al. Comparison of Long-Term Clinical Implications of Beta-Blockade in Patients With Obstructive Airway Diseases Exposed to Beta-Blockers With Different beta1-Adrenoreceptor Selectivity: An Italian Population-Based Cohort Study. Front. Pharmacol. 9, 1212 (2018).

13. Maltais, F. et al. Beta-Blockers in COPD: A Cohort Study From the TONADO Research Program. Chest 153, 1315-1325 (2018)

14. Garvin, J. H. et al. Exploration of ICD-9-CM coding of chronic disease within the Elixhauser Comorbidity Measure in patients with chronic heart failure. Perspect. Heal. Inf. Manag. 10, 1b (2013).

15. Greenland, S. \& Robins, J. M. Identifiability, exchangeability, and epidemiological confounding. Int. J. Epidemiol. 15, 413-419 (1986).

16. Walker, A. M. et al. A tool for assessing the feasibility of comparative effectiveness research. Comp. Eff. Res. 3, 11-20 (2013).

17. Bakris, G. L., Hart, P. \& Ritz, E. Beta blockers in the management of chronic kidney disease. Kidney Int. 70, 1905-1913 (2006).

18. Takeda, T. et al. Efficacy and safety of carvedilol in renal hypertension. A multicenter open trial. Eur. J. Clin. Pharmacol. 38(Suppl 2), S158-63 (1990).

19. Nowicki, M., Szewczyk-Seifert, G., Klimek, D. \& Kokot, F. Carvedilol does not modulate moderate exercise-induced hyperkalemia in hemodialysis patients. Clin. Nephrol. 57, 352-358 (2002).

20. DiNicolantonio, J. J. et al. beta-Blockers in hypertension, diabetes, heart failure and acute myocardial infarction: a review of the literature. Open Hear. 2, e000230 (2015).

21. Bakris, G. L. et al. Metabolic effects of carvedilol vs metoprolol in patients with type 2 diabetes mellitus and hypertension: a randomized controlled trial. JAMA 292, 2227-2236 (2004).

22. Liao, K.-M., Liang, F.-W. \& Li, C.-Y. Risks of all-cause and site-specific fractures among hospitalized patients with COPD. Medicine (Baltimore). 95, e5070-e5070 (2016).

23. Reid, I. R. et al. beta-Blocker use, BMD, and fractures in the study of osteoporotic fractures. J. Bone Miner. Res. 20, 613-618 (2005).

24. de Vries, F., Souverein, P. C., Cooper, C., Leufkens, H. G. M. \& van Staa, T. P. Use of beta-blockers and the risk of hip/femur fracture in the United Kingdom and The Netherlands. Calcif. Tissue Int. 80, 69-75 (2007).

25. Takeda, S. et al. Leptin regulates bone formation via the sympathetic nervous system. Cell 111, 305-317 (2002).

26. Patel, R. I. \& Beckett, R. D. Evaluation of resources for analyzing drug interactions. J. Med. Libr. Assoc. 104, 290-295 (2016).

27. Alatri, A. et al. Diagnosis and management of acute deep vein thrombosis: a joint consensus document from the European Society of Cardiology working groups of aorta and peripheral vascular diseases and pulmonary circulation and right ventricular function. Eur. Heart J. 39, 4208-4218 (2017).

28. Metra, M. et al. Beta-blocker therapy influences the hemodynamic response to inotropic agents in patients with heart failure: a randomized comparison of dobutamine and enoximone before and after chronic treatment with metoprolol or carvedilol. J. Am. Coll. Cardiol. 40, 1248-1258 (2002).

29. Bollano, E., Tang, M. S., Hjalmarson, A., Waagstein, F. \& Andersson, B. Different responses to dobutamine in the presence of carvedilol or metoprolol in patients with chronic heart failure. Heart 89, 621-624 (2003).

30. Dziedzic, T., Slowik, A., Pera, J. \& Szczudlik, A. Beta-blockers reduce the risk of early death in ischemic stroke. J. Neurol. Sci. 252, 53-56 (2007).

31. Savitz, S. I. et al. The novel beta-blocker, carvedilol, provides neuroprotection in transient focal stroke. J. Cereb. Blood Flow Metab. 20, 1197-1204 (2000) 
32. Lysko, P. G. et al. Neuroprotective effects of carvedilol, a new antihypertensive agent, in cultured rat cerebellar neurons and in gerbil global brain ischemia. Stroke 23, 1630-1636 (1992).

33. Remme, W. J. et al. Effect of carvedilol and metoprolol on the mode of death in patients with heart failure. Eur. J. Heart Fail. 9 , $1128-1135$ (2007).

34. Stoschitzky, K. et al. Differing beta-blocking effects of carvedilol and metoprolol. Eur. J. Heart Fail. 3, 343-349 (2001).

35. Stoschitzky, K., Stoschitzky, G., Brussee, H., Bonelli, C. \& Dobnig, H. Comparing beta-blocking effects of bisoprolol, carvedilol and nebivolol. Cardiology 106, 199-206 (2006).

36. Sessa, M. et al. Relationship Between Gender and the Effectiveness of Montelukast: An Italian/Danish Register-Based Retrospective Cohort Study. Front. Pharmacol. 9, 844 (2018).

37. Sessa, M. et al. Campania Preventability Assessment Committee (Italy): A Focus on the Preventability of Non-steroidal Antiinflammatory Drugs' Adverse Drug Reactions. Frontiers in Pharmacology 8, 305 (2017).

38. Huang, Y. L. et al. Impact of selective and nonselective beta-blockers on the risk of severe exacerbations in patients with COPD. Int. J. Chron. Obstruct. Pulmon. Dis. 12, 2987-2996 (2017).

39. Johnson, J. A. Ethnic differences in cardiovascular drug response: potential contribution of pharmacogenetics. Circulation 118, 1383-1393 (2008).

40. Italian National Institute of Statistics. [Cittadini Stranieri. Popolazione residente e bilancio demografico al 31 dicembre 2018 (dati provvisori) Comune: Caserta], https://www.istat.it/en/ (2019). Available at, http://demo.istat.it/str2018/query. php?Rip $=$ S4\&Reg $=$ R15\&Pro $=$ P061\&Com $=22 \& p a e s e=A 9999$. (Accessed: 8 th July 2019).

41. Parker, B. M., Rogers, S. L. \& Lymperopoulos, A. Clinical pharmacogenomics of carvedilol: the stereo-selective metabolism angle. Pharmacogenomics 19, 1089-1093 (2018).

42. Lymperopoulos, A., McCrink, K. A. \& Brill, A. Impact of CYP2D6 Genetic Variation on the Response of the Cardiovascular Patient to Carvedilol and Metoprolol. Curr. Drug Metab. 17, 30-36 (2015).

\section{Author Contributions}

Dr. Maurizio Sessa and Dr. Annamaria Mascolo wrote the paper and performed the data analysis. Prof. Morten Andersen, Prof. Annalisa Capuano and Prof. Francesco Rossi interpreted the results and wrote the papers. Dr. Maurizio Sessa, Dr. Annamaria Mascolo, Dr. Daniel Bech Rasmussen, Dr. Kristian Kragholm, Dr. Magnus Thorsten Jensen, Dr. Liberata Sportiello, Dr. Concetta Rafaniello, Mr. Giuseppe Michele Tari, Mrs Claudia Pagliaro, Prof. Morten Andersen, Prof. Francesco Rossi, and Prof. Annalisa Capuano commented on and approved the manuscript.

\section{Additional Information}

Competing Interests: Prof. Morten Andersen has participated in research projects funded by AstraZeneca, H. Lundbeck \& Mertz, Novartis, Pfizer and Janssen, with grants paid to the institutions where he has been employed, and has personally received fees from Medicademy, the Danish Pharmaceutical Industry Association, for leading and teaching pharmacoepidemiology courses. Other authors have no conflicts of interest that are directly relevant to the contents of this work.

Publisher's note: Springer Nature remains neutral with regard to jurisdictional claims in published maps and institutional affiliations.

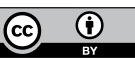

Open Access This article is licensed under a Creative Commons Attribution 4.0 International License, which permits use, sharing, adaptation, distribution and reproduction in any medium or format, as long as you give appropriate credit to the original author(s) and the source, provide a link to the Creative Commons license, and indicate if changes were made. The images or other third party material in this article are included in the article's Creative Commons license, unless indicated otherwise in a credit line to the material. If material is not included in the article's Creative Commons license and your intended use is not permitted by statutory regulation or exceeds the permitted use, you will need to obtain permission directly from the copyright holder. To view a copy of this license, visit http://creativecommons.org/licenses/by/4.0/.

(C) The Author(s) 2019 